\title{
Effects of loading frequency on cyclic parameters of unsaturated Zenoz kaolin
}

\author{
Mohsen Mojezi ${ }^{1, a}$, Mahnoosh Biglari ${ }^{2}$, and Mohammad Kazem Jafari ${ }^{1}$ \\ ${ }^{1}$ International Institute of Earthquake Engineering and Seismology (IIEES), Tehran, Iran \\ ${ }^{2}$ Razi University, Kermanshah, Iran
}

\begin{abstract}
According to the considerable effects of the loading frequency on the cyclic behaviour of the soils and lack of the sufficient and precise data in this field for the unsaturated soils, more attention needs to be paid to the experimental investigations in various loading paths and frequencies. Meanwhile the unsaturated cyclic tests require advanced equipment and need more precision and time. In this paper, the results of two groups of tests which were performed on an unsaturated fine grained soil in the same initial condition are presented and compared during specific stress paths (pre-cycles of loading and during cycles). The tests are performed using the same suction-controlled cyclic triaxial apparatus in the same stress path before loading cycles, but two conditions of the cyclic loading frequencies (slow cyclic tests with the constant suction condition during the cycles and rapid cyclic tests with the unsteady suction condition during the cyclic path). The results show that increase in the loading frequency leads to the considerable increase in the shear modulus of the samples in the same strain levels.
\end{abstract}

\section{Introduction}

The loading frequency (the cyclic loading rate) could be subjected to the machine foundation cases with variable loading rate. The fast cyclic loading rate can be unequalised in the suction distribution. Hence, understanding the effect of the loading rate is useful for the precise interpretation of the behaviour.

Several researches have been conducted for investigating the dynamic parameters of the unsaturated soils, especially small-strain shear stiffness (e. g. [1] to [8]).

In spite of the various studies on the cyclic and dynamic behaviours of the unsaturated materials in the small strain levels, the experimental data in the medium to large strain levels of the unsaturated materials are limited. For the first time, [9] studied the shear modulus and damping ratio of the unsaturated Zenoz kaolin materials using the new suction controlled triaxial apparatus at the medium to the large strains in a constant suction during the slow loading rate cycles. Since, the effects of cyclic loading frequency on the cyclic parameters are quite unexplored, recently, [10] studied the cyclic parameters by the same apparatus on the same material at the higher cyclic rate. In this paper the results of two groups of the tests are compared. These tests have been performed on the unsaturated Zenoz kaolin under the identical stress paths including (i) equalization (ii) isotropic consolidation (iii) slow or fast rate cyclic loading.

\section{Testing Apparatus and materials}

In this research the suction-controlled cyclic triaxial apparatus developed by [9] in International Institute of Earthquake Engineering and Seismology (IIEES) was used (Figure1) (More detailed data about the apparatus could be found in [9]). Suction is applied and controlled by Axis Translation Technique via HAVEdiscs from top and bottom of the specimens in suction controlled triaxial cyclic device.

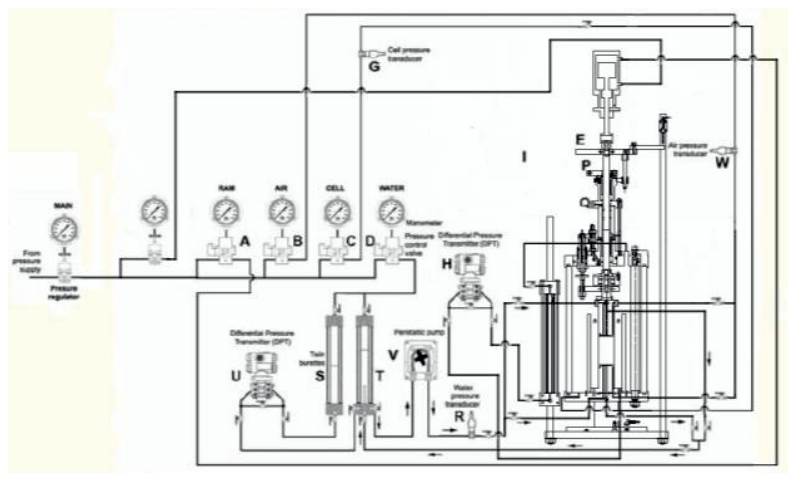

Figure 1. Suction controlled cyclic triaxial device [9]

In this research Zenoz kaolin which is a commercial finegrained soil was studied in the unsaturated condition. The physical specifications of this soil are presented in Table 1.

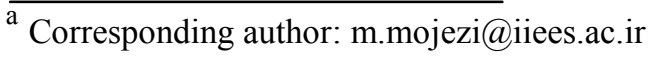


Table 1. Physical specification of the tested soil

\begin{tabular}{|l|c|}
\hline Classification (USCS) & CL \\
\hline Clay Fraction (\%) & 18 \\
\hline Silt Fraction (\%) & 60 \\
\hline Plasticity Index: PI (\%) & 12 \\
\hline Liquid Limit: LL (\%) & 29 \\
\hline Plastic Limit: PL (\%) & 17 \\
\hline Specific Gravity: $\mathrm{G}_{\mathrm{S}}$ & 2.65 \\
\hline Optimum Water Content: $\omega_{\text {opt }}(\%)$ & 15.4 \\
\hline Maximum Dry Unit Weight: $\gamma_{\mathrm{d}(\mathrm{max})}\left(\mathrm{g} / \mathrm{cm}^{3}\right)$ & 1.74 \\
\hline Initial Void Ratio: $\mathrm{e}_{0}$ & 1.19 \\
\hline Initial Degree of Saturation: $\mathrm{Sr}_{0}(\%)$ & 27 \\
\hline
\end{tabular}

To achieve the normally consolidated condition, the samples were compacted with $11.9 \%$ of water content (3.5\% less than the optimum water content) and dry unit weight of $12 \mathrm{kN} / \mathrm{m} 3$ (69\% of the maximum dry unit weight, obtained by the standard proctor test).The specimens were compacted using under compaction method proposed by [11], in seven layers with diameter of $38 \mathrm{~mm}$ and final height of $76 \mathrm{~mm}$.

\section{Stress Paths}

In the both series of tests, the samples were equalized in the suction levels of 0,150 and $300 \mathrm{kPa}$, and then were isotropically consolidated up to the mean net stress of $100 \mathrm{kPa}$. Finally, the specimens were subjected to twoway deviatoric cyclic loading of 18,42 and $81 \mathrm{kPa}$ in each suction level. In the slow group of the tests (i.e. [9]), in order to keep the suction condition constant during the loading cycles, the cycles were performed with very low rate $(8 \mathrm{kPa} / \mathrm{hr}$ : i.e. $3.09 \mathrm{E}-5 \mathrm{~Hz}$ for the deviatoric stress amplitude of $18 \mathrm{kPa}, 1.32 \mathrm{E}-5$ for the deviatoric stress amplitude of $42 \mathrm{kPa}$, and $6.86 \mathrm{E}-6$ for the deviatoric stress amplitude of $81 \mathrm{kPa}$ ). In the higher cyclic loading rate group of the tests $([10])$ the cycles were done with the frequency of $0.003 \mathrm{~Hz}$ while the water content was kept constant (consequently, the suction is not constant during the cyclic loading).

The stress paths of the both groups of the performed tests are schematically plotted in Figure 2 . In this paper the tests are named as a term like "S-number-D-number", in which the number after "S" refers to the suction level and the number after "D" refers to the deviatoric stress amplitude.

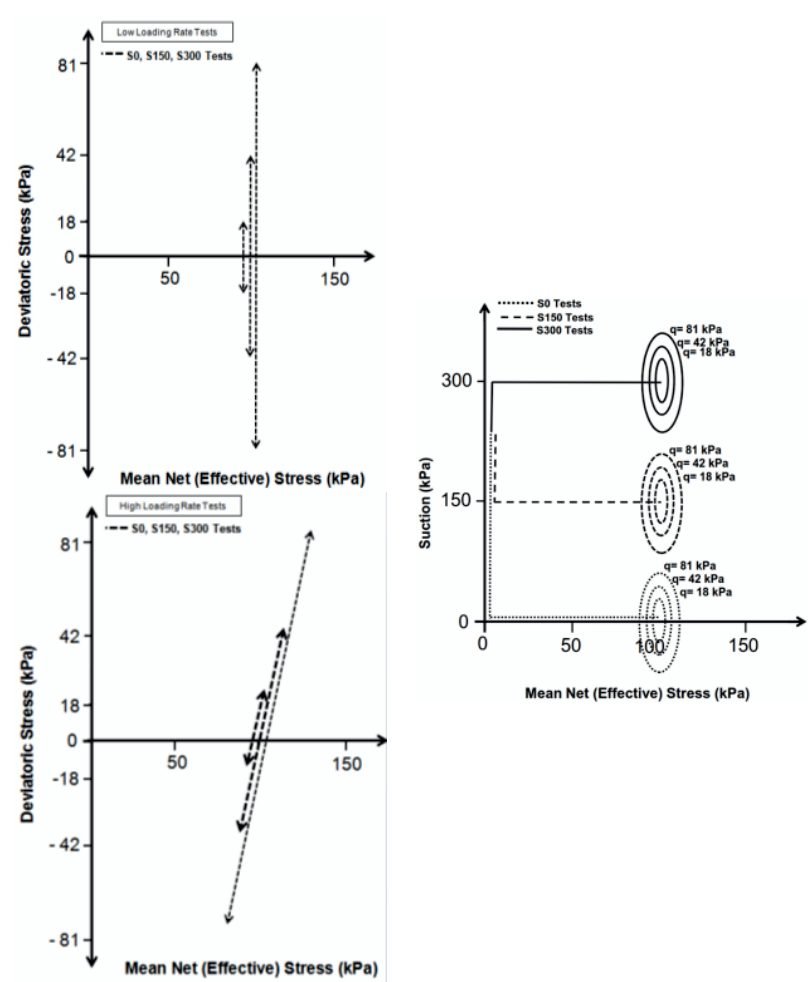

Figure 2. Stress paths of the low and high cyclic rate tests

In the slow rate tests, the loading speed was kept in a way that the suction equalization condition of the samples was not changed during all of the stress paths.

In the high rate tests, however, the suction equalization condition is not maintained during the cyclic loading(due to higher loading frequency), hence a separate sample with the same pre-cyclic condition was tested for each amplitude of the deviatoric stresses (i.e.18, 42 and 81 $\mathrm{kPa}$ ) and each suction levels (i.e. 0, 150 and $300 \mathrm{kPa}$ ).

\section{Results}

Following the changes of two parameters, specific volume $(\mathrm{v}=1+\mathrm{e})$ and specific water volume $\left(\mathrm{v}_{\mathrm{w}}=1+\mathrm{S}_{\mathrm{r}} \cdot \mathrm{e}\right)$, are presented versus time and mean net stress $\left(\mathrm{p}-\mathrm{u}_{\mathrm{a}}\right)$.

Figures 3 and 4 show the specific water volume changes $\left(\mathrm{dv}_{\mathrm{w}}\right)$ versus time for the tests for the suction of $150 \mathrm{kPa}$ and $300 \mathrm{kPa}$ in the slow and high rate groups during the initial suction equalization process, respectively. According to these results, in both groups, the samples which were subjected to the suction level of $150 \mathrm{kPa}$ absorbed water whereas the samples with the suction level of $300 \mathrm{kPa}$ lost water. It means that the compacted specimen has initial suction level higher than $150 \mathrm{kPa}$ and lower than $300 \mathrm{kPa}$. Initial suction of these ideal samples were measured by [6] at $240 \mathrm{kPa}$. 


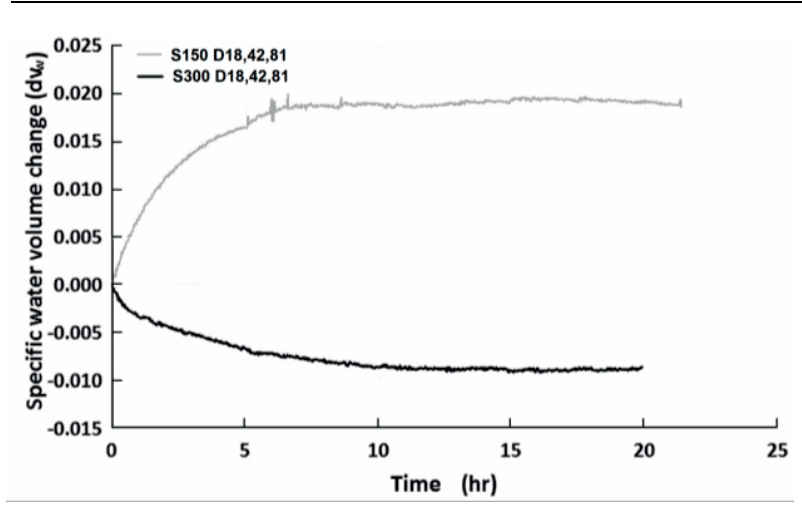

Figure 3. Water volume changes $\left(\mathrm{dv}_{\mathrm{w}}\right)$ during initial suction equalization process, in the slow rate tests [9]

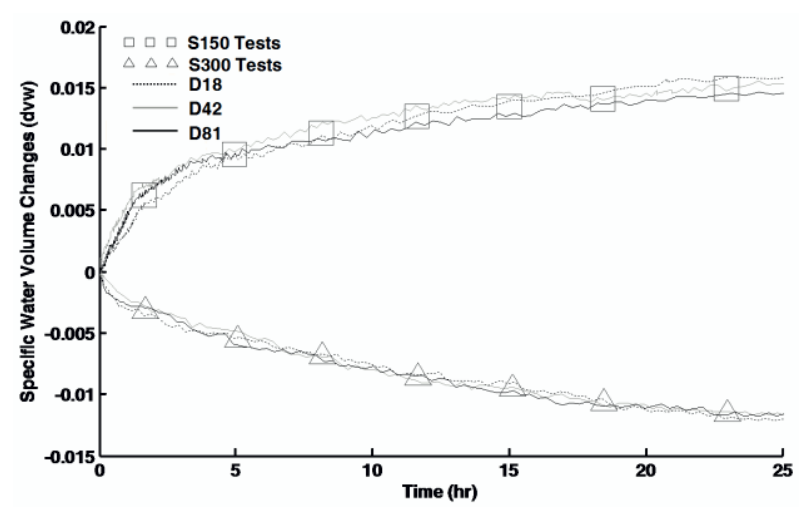

Figure 4. Water volume changes $\left(\mathrm{dv}_{\mathrm{w}}\right)$ during initial suction equalization process, in the high rate tests [10]

In Figures 5 and 6 the specific volume changes (dv) of the samples are plotted versus the time in the suction levels of $150 \mathrm{kPa}$ and $300 \mathrm{kPa}$ for the slow and the high rate tests, respectively. As seen in these figures all of the samples lost the volume due to compaction for the suction level of $300 \mathrm{kPa}$ and collapse for the suction level of $150 \mathrm{kPa}$ during the equalization process.

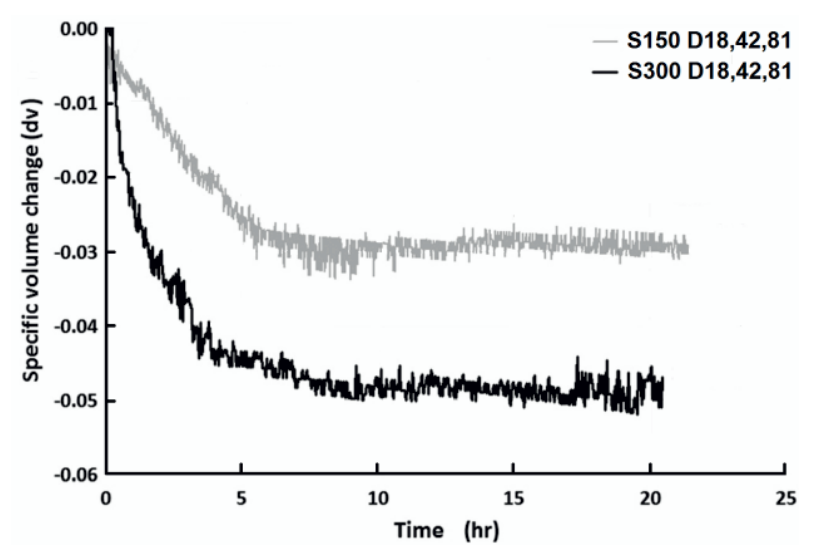

Figure 5. Specific volume changes $(\mathrm{dv})$ versus time in the slow rate tests [9]

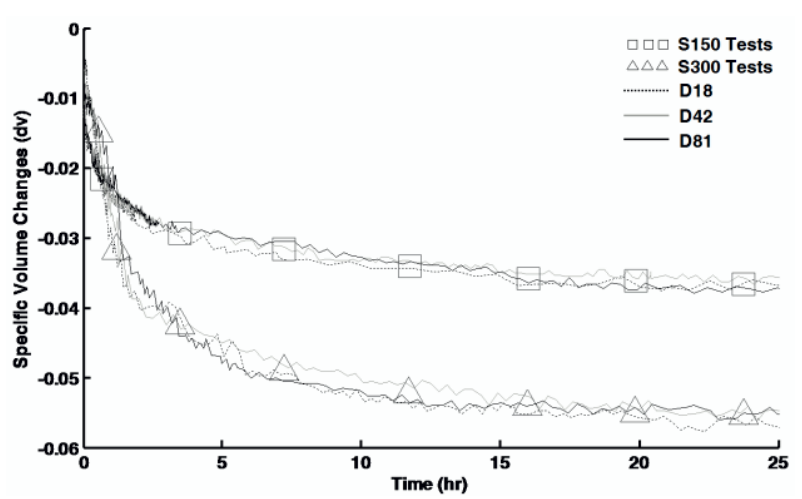

Figure 6. Specific volume changes (dv) versus time in the fast rate tests [10]

The specific volume variations of the specimens $(v=1+e)$ versus the mean net stress $\left(p-u_{a}\right)$ are presented in Figures 7 and 8 for the slow and the high rate tests, respectively.

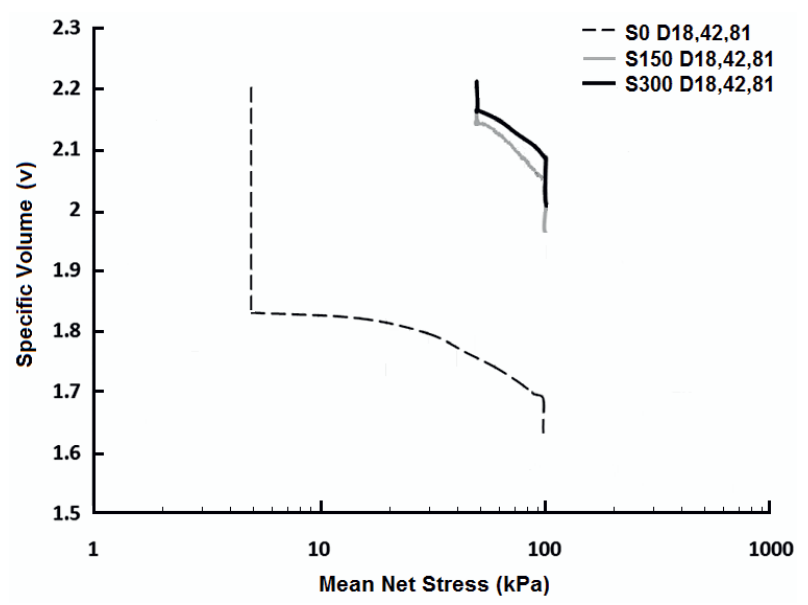

Figure 7. Specific volume (v) versus Mean net stress, during the slow tests [9]

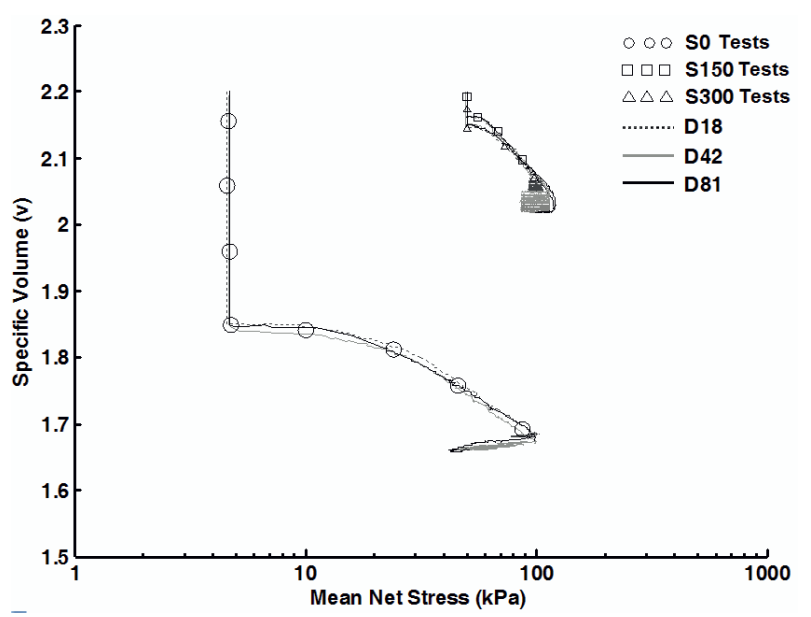

Figure 8. Specific volume (v) versus Mean net stress, during the fast tests

In both test groups, after the mean net stresses of 100 $\mathrm{kPa}$, the cyclic deviatoric stress loading was applied up to the specific amplitudes of 18,42 , and $81 \mathrm{kPa}$. The cyclic loadings were applied with the rate of $8 \mathrm{kPa} / \mathrm{hr}$ in the 
slow rate group and the frequency of $0.003 \mathrm{~Hz}$ in the high rate tests. In the slow rate group due to very time consuming (e. g. almost 40 hours for the deviatoric amplitude of $81 \mathrm{kPa} / \mathrm{hr}$ ), 5 cycles were performed but in the high rate tests the samples were subjected to 40 cycles in each amplitude of the deviatoric stress for each suction level. Although in some of the high rate tests due to the failure which were occurred for the samples during the cyclic loading (especially for the higher amplitudes), which the tests were stopped in the less cycle numbers.

The compatibility of the volumetric results during the equalization and the isotropic compaction process show that the specimens in the both groups are identical. At deviatoric cyclic loading phase, due to increase in the pore water pressure in the high rate test group, similar to the saturated liquefaction evaluation tests the mean net stress decrease slightly in the zero suction levels.

The variations of the specific water volume $\left(v_{w}\right)$ of the samples versus the mean net stress $\left(p-u_{a}\right)$ are shown in Figure 9 for the slow rate tests and in Figure 10 for the higher rate tests. In the zero suction level tests (saturated tests), $\mathrm{v}_{\mathrm{w}}$ was increased during equalization process due to the larger amount of the water which were entered the samples and then decreased due to the isotropic consolidation process. In the tests with suction level of $150 \mathrm{kPa}$, the samples were absorbed a little amount of water and vw was slightly increased up to achieve the constant suction of $150 \mathrm{kPa}$ and was approximately remained constant. For $300 \mathrm{kPa}$ suction the trend is reverse which is happened due to the increase in the initial suction level.

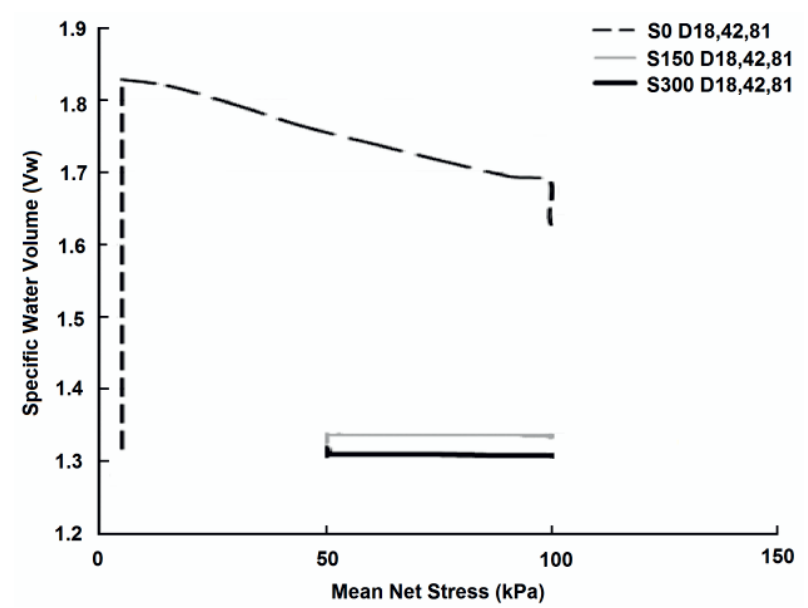

Figure 9. Variation of the specific water volume versus the mean net stress in the slow rate tests [9]

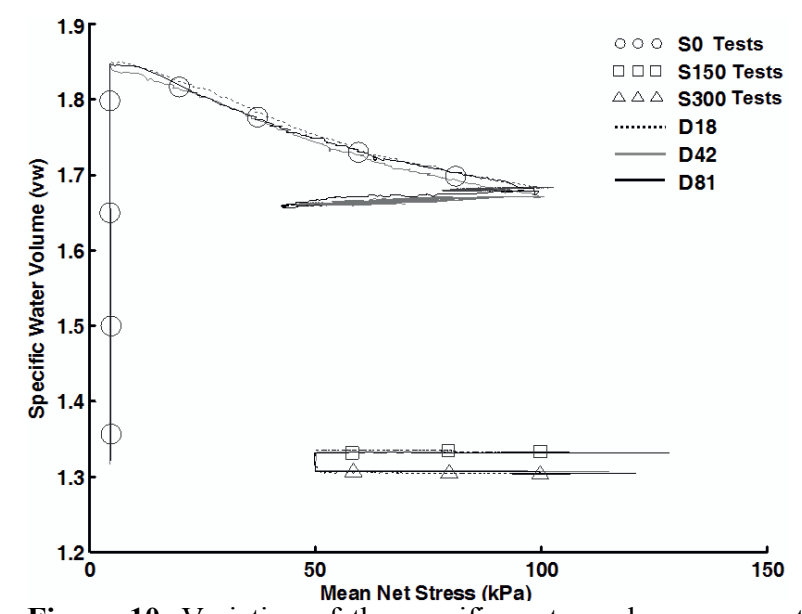

Figure 10. Variation of the specific water volume versus the mean net stress in the fast rate tests

The variations of the shear modulus $(G)$ versus the shear strain are presented in Figures 11 and 12 for the slow rate and the high rate tests, respectively. The variations of the damping ratio (D) for the slow and the high cyclic loadings are shown in Figures 13 and 14, respectively. The values related to the slow rate tests are presented for $1,2,3,4,5$ cycle numbers and the results of the high rate tests are presented for $1,3,5,10,15,20,25,30,35,40$ cycle numbers in the various suction levels and the various deviatoric stress amplitudes. The values of the first cycle are connected to each other by the dotted line for the saturated tests, the grey line for the suction level of $150 \mathrm{kPa}$, and the black line for the suction level of 300 $\mathrm{kPa}$.

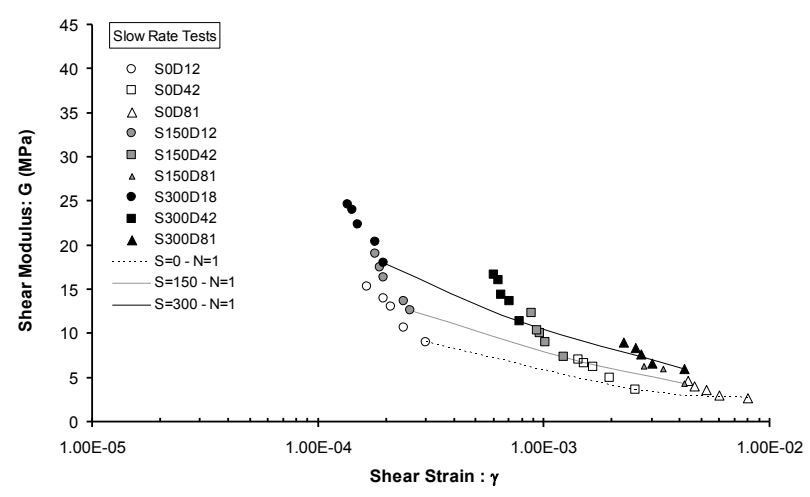

Figure 11. Variations of the shear modulus $(\mathrm{G})$ versus the shear strain in the slow rate tests [9]

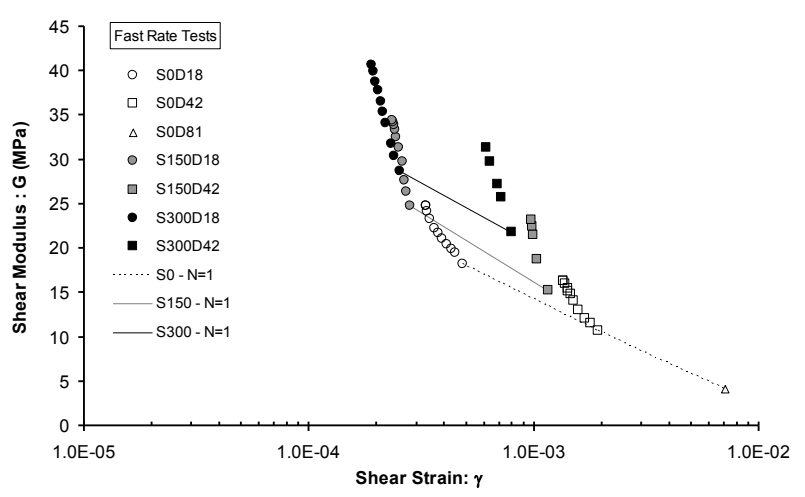

Figure 12. Variations of the shear modulus $(\mathrm{G})$ versus the shear strain in the fast rate tests [10] 


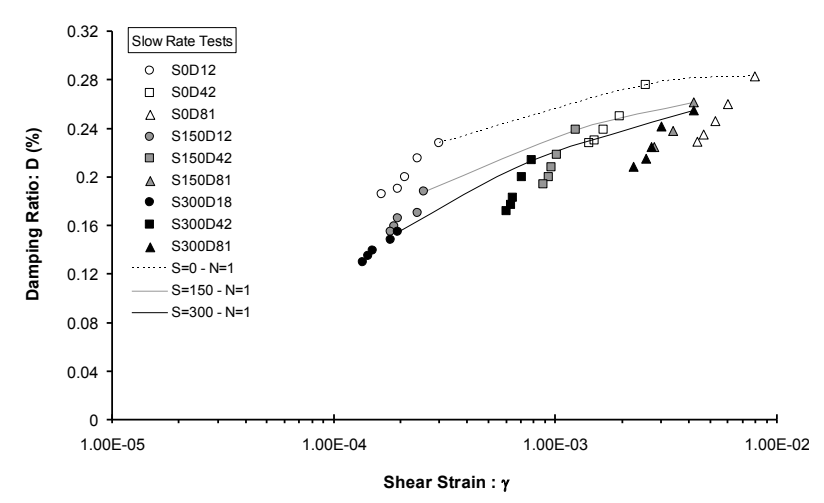

Figure 13. Variations of the damping ratio (D) versus the shear strain in the slow rate tests [9]

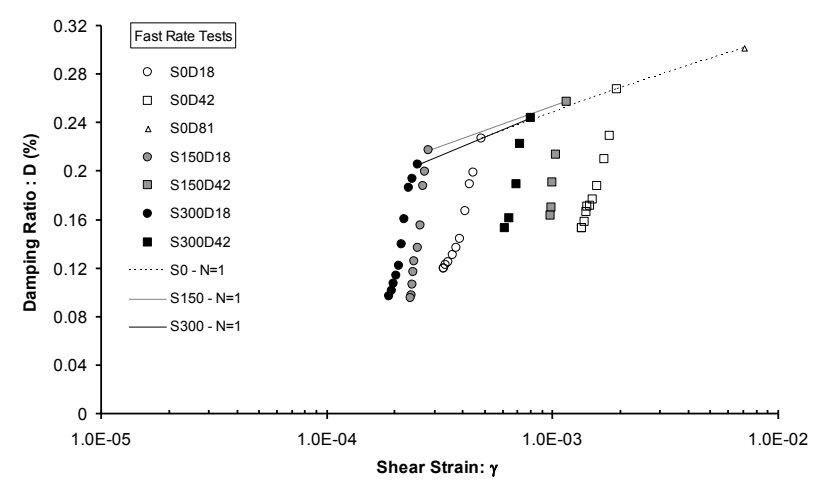

Figure 14. Variations of the damping ratio (D) versus the shear strain in the fast rate tests [10]

According to the Figures 11 and 12, in the same strain level and cycle's number, the increase in the suction level has been led to the increase in the shear modulus. On the contrary, Figures 13 and 14 show decrease in the damping ratio due to the increase in the suction level in same strain level and cycle's number.

Also, the increase in the number of cycles in all of the suction levels induces the sample stiffness and is followed by the shear modulus increase and the damping ratio decrease. This issue is similar for both groups of the tests but the trend is sharper in the higher rate tests.

On the other hand, the comparison between the presented results in Figures 11 and 12 is shown that in the same strain levels, the shear modulus of the high rate tests significantly are more than the slow ones. However, the increase in the rate of the cyclic loading could has led to this increase, it would be happened by uncontrolled variation in the suction due to the high rate of the cyclic loading.

Meanwhile, the results of the damping ratio in the both groups of the tests do not show a considerable different.

\section{Conclusions}

The effects of the loading rate on the cyclic parameters of the unsaturated Zenoz kaolin are studied.

The results showed the increase in the suction level has been led to increase in the shear modulus and decrease in the damping ratio due to the virtual cohesion which has been induced by the suction. Furthermore, increase in the cycle's number has been resulted in the shear modulus increase and the damping ratio decrease for all of the suction levels and loading rates.

In summary, the cyclic loading rate variation were significantly changed the cyclic parameters of the fine grained unsaturated soils. Hence, in the same suction level and equal strain, the shear modulus of the higher rate tests were more than the slow ones. Meanwhile, there were not observed considerable differences in damping ratio. Hence, the loading frequency effects need more consideration in modelling of the cyclic behaviour of unsaturated.

\section{References}

1. EAM. Marinho, R.J. Chandler, M.S. Crilly. The 1st Intern. Conf. on Unsat. Soils, Paris; p. 535-539 (1995).

2. C. Mancuso, R. Vassallo, A. d'Onofrio. Can Geotech J; 39(1):22-31 (2002).

3. R. Vassallo, C. Mancuso, F. Vinale. Can Geotech J; 44(4):447-462 (2007).

4. C.W.W. Ng, S.Y. Yung. Géotechnique; 58(1):23-35 (2008).

5. M. Biglari, C. Mancuso, A. d'Onofrio, M.K. Jafari, A. Shafiee. Comput. and Geotech.; 38(5): 709-720 (2011).

6. M. Biglari, A. d'Onofrio, C. Mancuso, M.K. Jafari, A. Shafiee, I. Ashayeri. Can. Geotech. J.; 49(3): 311-322 (2012).

7. A. Heitor, B. Indraratna, C. Rujikiatkamjorn. Can. Geotech. J.; 50(2):179-188 (2013).

8. K.S. Wong, D. Mašín, C.W.W. Ng. Comput. and Geotech.; 56:28-39 (2014).

9. M. Biglari, M.K. Jafari, A. Shafiee, C. Mancuso, A. d'Onofrio. Geotech. Test. J; 34(5):525-536 (2011).

10. M. Mojezi, M.K. Jafari, M. Biglari, Int. J. Civil Eng.; 13(3) (Transaction B: Geotech. Eng.)(2015)

11. R.S. Ladd. Geotech. Test. J.;; 1(1):16-23 (1978). 